\title{
Cardiac rhabdomyomata as a marker for the antenatal detection of tuberous sclerosis
}

\author{
D C CRAWFORD, C GARRETT, M TYNAN, B G NEVILLE, AND L D ALLAN \\ Departments of Paediatrics and Medical Genetics, Guy's Hospital, London SEI 9RT.
}

SUMMARY We report the echocardiographic identification of cardiac tumours in antenatal life in a pregnancy where the father was known to have tuberous sclerosis This allowed termination of an affected pregnancy in the second trimester.

Tuberous sclerosis is an autosomal dominant condition of varying penetrance, in which tuberose deposits are found mainly in the brain but also in the heart, kidneys, and lungs. The associated neurological problems of epilepsy, mental retardation, and occasional motor deficit are caused by the presence of tubers and other defects in cerebral development. The tubers become calcified and are detectable on computerised tomographic scanning of the head. Cardiac manifestations of the disease include multiple rhabdomyomata, which may cause arrhythmias and sudden death, or be large enough to be obstructive. ${ }^{12}$ Frequently cardiac, renal, and lung lesions are asymptomatic and discovered at necropsy. There are also skin manifestations of the disease in the form of shagreen patches or adenoma sebaceum.

We report the echocardiographic identification of cardiac tumours in antenatal life in a pregnancy where the father was known to have tuberous sclerosis. This allowed termination of an affected pregnancy in the second trimester.

\section{Family case report}

The proband presented on the first day of life with signs of cyanotic congenital heart disease. Crosssectional echocardiography demonstrated a tumour completely obstructing the right ventricular cavity and multiple tumourous deposits in the left ventricle. Partial resection of the obstructive tumour was attempted but the child died. Necropsy confirmed the echocardiographic findings of rhabdomyomata and also demonstrated the typical intracranial

Received for publication 1 February 1983.

Accepted for publication 19 February 1983. lesions associated with tuberous sclerosis. The rest of the family was then investigated. The elder sib had epilepsy, severe mental retardation, adenoma sebaceum, and a shagreen patch. Periventricular calcification and dilation of the left lateral ventricle was evident on computerised tomography of the head. Cross-sectional echocardiography demonstrated the presence of multiple rhabdomyomata of the heart which were small and not obstructive. The father had had epilepsy in childhood and made slow progress at a normal school. He had obvious adenoma sebaceum and CT scanning showed

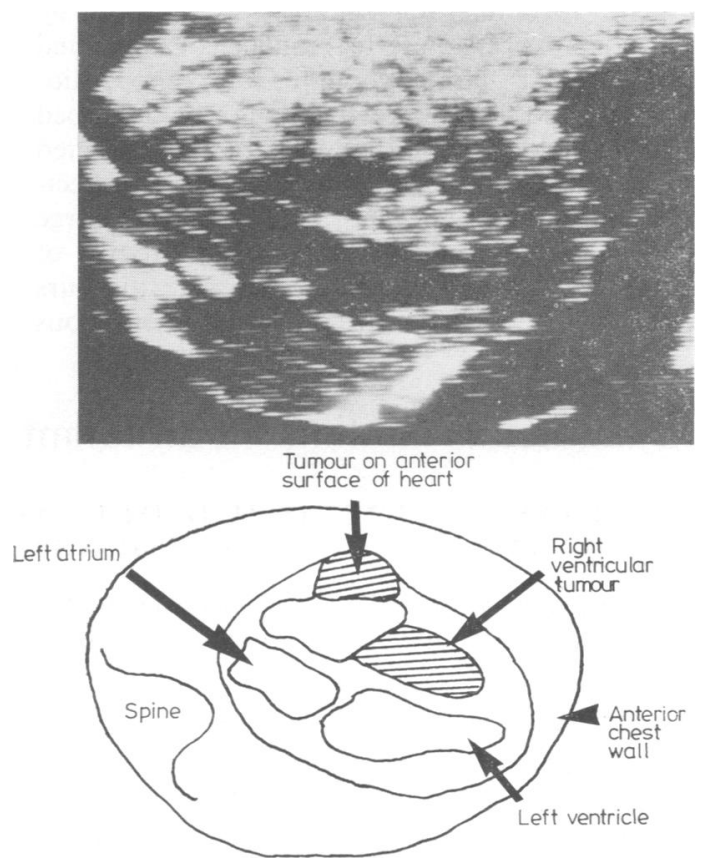

FIGURE Echocardiogram of the fetal heart during the 22nd week of pregnancy. A large tumour is seen within the body of the right ventricle and another tumour on the anterior surface of the right atrium. 
typical periventricular calcified lesions. No cardiac tumours were visible echocardiographically.

The parents were counselled that a future child was at a 1 in 2 risk of being affected by tuberous sclerosis, but that the manifestations of this were very variable. However, the mother unintentionally became pregnant soon afterwards and was offered fetal cardiac ultrasonography as a possible method of antenatal prediction of an affected child. Fetal echocardiography was performed on two occasions during the second trimester. The fetal heart was examined initially at 18 weeks' gestation and appeared normal. A second examination of the fetal heart during the 22nd week of pregnancy revealed the presence of a large tumour within the body of the right ventricle and at least one tumour on the surface of the heart (figure). As this fetus was almost certainly affected by tuberous sclerosis, termination of the pregnancy was offered. Necropsy confirmed the presence of a rhabdomyoma filling the body of the right ventricle with multiple smaller tumours in the left ventricle and on the external surfaces of the heart.

\section{Discussion}

The gene for tuberous sclerosis is notoriously variable in its expression. The frequency of associated rhabdomyomata large enough for echocardiographic detection is unknown. The family described may be unusual in that three out of four affected family members had echocardiographically detectable cardiac tumours. The tumours were large enough to cause circulatory obstruction in two of the four. Finding no evidence of cardiac tumours would not have excluded the diagnosis of tuberous sclerosis in this case, but the positive identification of a cardiac tumour indicated a third affected child $\overrightarrow{\bar{N}}$ in this family. Even in the absence of a family $\overrightarrow{0}$ history, a cardiac tumour in any patient found $\frac{C}{0}$ histologically to be a rhabdomyoma implies the $\overline{\frac{\sigma}{\sigma}}$ diagnosis of tuberous sclerosis whether that disease $\vec{\nabla}$ is clinically manifest or not. ${ }^{3}$

The fetal heart can be well visualised echocardio- $ळ$ graphically between 16 weeks' gestation and term. ${ }^{4} \overrightarrow{0}$

As this case shows, cardiac tumours can be $\overrightarrow{-}$ detected as a marker for tuberous sclerosis. As the $\bar{\omega}$ technique gives rise to no fetal or maternal discomfort it should be offered for possible antenatal diagnosis in a pregnancy known to be at risk, i although the absence of visible cardiac lesions ${ }_{-}$ would not exclude the presence of the disease. It is $\dot{\omega}$ possible that in the future detailed ultrasonography $\omega$

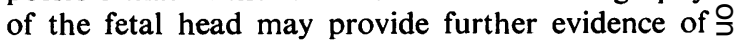
the disease when it is present.

\section{References}

1 Bohm N, Krebs G. Solitary rhabdomyoma of the heart: clinically silent case with sudden, unexpected death in an $\infty$ 11-month-old boy. Eur J Pediatr 1980;134:167-73.

2 Allan LD, Joseph MC, Tynan M. Clinical value of echocardiographic colour image processing in two cases of primary cardiac tumour. Br Heart $J 1983 ; 49: 154-6$.

3 Davies MJ. Tumours of the heart and pericardium. In: Pomerance A, Davies MJ, eds. The pathology of the heart. London: Blackwell, 1975:423-40.

4 Allan LD, Tynan M, Campbell S, Anderson RH. Normal $\varrho$ fetal cardiac anatomy -a basis for the echocardio- $\overrightarrow{\bar{A}}$ graphic detection of abnormalities. Prenatal Diagnosis $\frac{0}{3}$ $1981 ; 1: 131-9$.

Correspondence and requests for reprints to Dr D C Crawford, Department of Paediatrics, Guy's Hospital, London SE1 9RT.

\title{
Unusual ocular findings in an infant with cri-du-chat syndrome
}

\author{
SOFIA KitSiOU-TZELI*, H D DELLAGRAMMATICAS*, C B PAPAS*, \\ I D LADAS †, AND C S BARTSOCAS* \\ *Second Department of Pediatrics, University of Athens, 'P and A Kyriakou' Children's Hospital; and \\ $\dagger$ Department of Ophthalmology, University of Athens, Greece.
}

SUMMARY A newborn male with cri-du-chat syndrome, congenital nuclear cataracts, microspherophakia, and probably ectopic lenses is reported. Microspherophakia in cri-du-chat syndrome has not been previously described. The congenital cataracts were inherited from

Received for publication 27 September 1982. Accepted for publication 8 November 1982. his mother who had a balanced 5;13 trans? location; the two events are considered to be coincidental and a possible 'position effect' was excluded, since the other members of her family" with congenital cataracts, were chromosomallyo normal. This is the fourth case reported where familial cri-du-chat syndrome involves chromo-市 somes $5 p$ and $13 q$. 